\title{
A Jacobi-based Algorithm for Computing Symmetric Eigenvalues and Eigenvectors in a Two-dimensional Mesh
}

\author{
Dolors Royo. Miguel Valero-García, and Antonı González \\ Departament d'Arqutectura de Computadors \\ Universitat Politècnica de Catalunya \\ c/ Jord Girona 1-3, Campus Nord - Edifici D6 \\ E-08034 Barcelonal (Spain) \\ Phone. +34-3-401 7189 \\ E-mail: \{dolors, miguel, antonio\} (a) ac.upc es
}

\begin{abstract}
This paper proposes an algorthm for computing symmetric eigenvalues and eigenvectors that uses a onesided Jacobi approach and is targeted to a multicomputer in which nodes can be arranged as a two-dimensional mesh with an arbitrary number of rows and columns. The algorithm is analysed through simple analytical models of execution time, which show that an adequate choice of the mesh configuration (number of rows and columns) can improve performance significantly, with respect to a onedimensional configuration, which is the most frequently considered scenario in current proposals. This improvement is especially notuceable in large systems.'
\end{abstract}

\section{Introduction}

Many authors have focused on the problem of computing the eigenvalues and eigenvectors of a real symmetric matrix on different types of computing machines, including systolic arrays (i.e. [2], [6]) shared memory multiprocessors (i.e. [1]) and distributed memory multiprocessors (multicomputers, for short) (i.e. [4]) Those proposals in which the target system is a meshconnected multicomputer are particularly relevant, since this type of parallel machine organization is considered to be a promising platform for large scale efficient supercomputing.

In this paper. the target system is a $2^{d}$-node wormhole multicomputer, arranged as a $2^{\prime} \times 2^{\prime}$ two-dimensional fourneighbour mesh, with any value for $r$ and $c$, provided that $r+c=d$. Therefore, the results of this paper are useful in the context of application specific mesh design or in the context of multicomputers which allow such an arbitrary configuration of the nodes, as it is the case of transputerbased systems. Additionally, the results of the paper are useful as a first step to solve the problem of eigenvalue and eigenvector computation on a multicomputer with a

I. This work was supported by the Ministry of Education and Science of Spain (CICYT TIC-429/95) fixed configuration of nodes (i.e a squared two or three dimensional mesh), which is the subject of further work [8]

Proposals for parallel ergenvalue and eigenvector computation are firequently based on Jacobi methods because, although they require more arithmetic computations than others (e.g. QR iteration), they exhibit a large degree of potential parallelism. The one-sided Jacobi method [3] is particularly attractive since it has lower communication requrements than the alternative two-sided method [10]

Several approaches have been proposed for the parallel organization of the computations in a Jacobi method. Such approaches are referred to as Jacobi orderngs A particular organization of a Jacobi ordering in the form of a set of cooperating processes (or nodes) is referred to as a Jacobi algorithm.

Parallel Jacobi algorithms for eigenvalue computation on mesh multicomputers known to us use a onedimensional organization of the nodes, either with a wraparound link ( $\mathrm{e}$ rungs) or without it (i.e. lines). For this reason, they will be called one-dimensional algorithms. See $[4|| ,2|| 9$,$] for a few relevant proposals of$ one-dimensional algorithms.

This paper proposes a novel Jacobi algorithm that uses a two-dimensional organization of the nodes and will be called the two-dimensional algorithm. The algorithm uses the one-sided Jacobi method and a Jacobi ordering used in a one-dimensional algorithm proposed in 14]. We show that the two-dimensional algorithm is more efficient on the target system than the one-dimensional algorithm, since it has a lower communication cost. This will be shown through simple analytical models of performance, which enable us to derive the number of rows $\left(2^{\prime}\right)$ and columns $\left(2^{c}\right)$ that minimize the execution time on the configurable mesh.

The icleas brought together in this paper can be used to derive other two-dimensional algorithms which use alternative Jacobi orderings. Additionally, our proposal can also be used for singular value decomposition (SVD) snce Jacobr methods can also be applied in this matrix 
computation [11].

The rest of the paper is organized as follows: Section 2 describes the target system, the problem, and the onedimensional algorithm that is used for comparison purposes. Section 3 describes the proposed twodimensional algorithm. Section 4 develops analytical models of execution time which are used to evaluate the proposal. Finally, section 5 summarizes the main conclusions of this work.

\section{Background}

\subsection{Target architecture}

Consider a multicomputer system with $2^{d}$ nodes that can be arranged as a $2^{r} \times 2^{c}$ two-dimensional (2D) fourneighbour mesh (neighbours in the north, east, west and south directions), with any value for $r$ and $c$, provided that $r+c=d$. We assume full-duplex links between nodes and a communication system that uses the wormhole switching model [7]. In this model, the header of the message is sent through the network to establish a path between the source and the destination. Then, the rest of the message is sent in a pipeline fashion. The conclusions of this work can be readily extended to systems with circuit switching or virtual cut-through routing. This is because the differences among these models, which are found in the strategies to establish the path or to solve a conflict in the links or buffers, are relevant neither to the proposed algorithms nor the evaluation assumptions.

A one-port model is assumed with regard to the node organization [7]. In this model, every node can send/receive only one message to/from the network, at the same time. Finally, we assume a synchronous communication model. In other words, a sending node is blocked until the message has been completely received by the destination node.

For algorithm modelling purposes, we assume that nodes spend a time $t_{\text {c }}$ to perform a floating-point computation, and a time $t_{\mathrm{s}}+N \times t_{\rho}$ to send a message of $N$ floating point numbers (as is common, in a wormhole system, the time required to establish the path between the sending and receiving nodes is neglected in comparison to the startup time $t_{\mathrm{s}}$ and the transmission time $N \times t_{e}$ ).

\subsection{Jacobi methods for symmetric eigenvalue and eigenvector computation}

Let $A$ be a $m \times m$ real symmetric matrix, $\lambda$ is an eigenvalue of $A$ if there exists a vector $x$ such that.

$$
A x=\lambda x
$$

Vector $x$ is the eigenvector associated with eigenvalue $\lambda$. A Jacobi method for eigenvalue and eigenvector computation is an iteratıve procedure aimed at reducing $A$ to a diagonal form by applying a series of similarity transformations. Since similarity transformations preserve the eigenvalues, the elements in the diagonal of the resulting matrix are the eigenvalues of $A$.

Plane rotations are used to build every similarity transformation [5]. Specifically, in a given iteration $k$ of the algorithm, a plane rotation $R_{k}$ is used to zero one pair of off-diagonal symmetric element. by applying a simularity transformation as follows

$$
A_{k+1}=R_{k}^{\mathrm{T}} \times A_{k} \times R_{k} \text { for } k=0,1,2, \ldots \text { and } A_{0}=A
$$

The plane rotation $R_{k}$ requrred to zero elements $A_{k}(p, q)$ and $A_{h}(q, p)$ is built upon the values $s=\sin \left(\alpha_{p, q}\right)$ and $c=\cos \left(\alpha_{p, q}\right)$, being $\alpha_{p, q}$ the rotation angle computed from $A_{k}(p, q), A_{k}(p, p)$ and $A_{h}(q, q)$ as described in $[5]^{p}$.

A total of $m(m-1) / 2$ distinct transformations are required to zero all the off-diagonal elements exactly once. This collection of transformations is called a sweep. The Jacobi method is iterative because a transformation may fill elements that had been zeroed in previous transformations and therefore several sweeps are required so that $A$ approaches a diagonal form.

The convergence rate of the algorithm depends on the ordering in which the transformations are applied in one sweep. The classic Jacobi ordering zeroes the off-diagonal elements in the order determined by their absolute value, from the highest to the lowest. When using this ordering, the algorthm converges very quickly, but spends most of the time searching for the next element to be zeroed. In the cyclic Jacobi orderng, the off-diagonal elements are zeroed according to a predetermined ordering (i.e., by rows or by columns) Even though the cyclic Jacobi ordering needs more sweeps to converge, it is in general faster than the classic ordering since every sweep has a lower cost. Finally, when required, the eigenvectors are obtained from the columns of matrix $R_{0} \times R_{1} \times R_{2} \times \ldots$

The method clescribed so far is referred to as the twosided Jacobi method. snce in iteration $k$ matrix $A_{k}$ is multiplied from the lett (by $R_{k}^{\mathrm{T}}$ ) and from the right (by $R_{h}$ ), involving row and column update. Due to this feature, the two-sided method incurs a high communication cost when implemented on a multicomputer, where matrices are distributed among the nodes.

As an alternative to the two-sided method, the onesided method organizes the computations in such a way that only column updates are required. Specifically, the one-sided method organizes the computations as follows [3]:

$$
\begin{gathered}
\bar{A}_{j}=A \text { and } U_{0}=I \\
\bar{A}_{k+1}=\bar{A}_{k} \times R_{k} \text { and } U_{k+1}=U_{k} \times R_{k} \text { for } k=0,1,2, \ldots \\
\text { Since } A_{k}=R_{h}^{\mathrm{T}} \times \bar{A}_{k} \text {, the values of } A_{k}(p, q), A_{k}(p, p) \text { and }
\end{gathered}
$$
$A_{\Lambda}(q, q)$. which are required in iteration $k$ to compute the rotation angle $\alpha_{p}$ a used to build $R_{k}$, are recovered from $\bar{A}_{k}$ as follows:

I From now on. $R$ will be used to denote either the rotation matu1 (t) the $-1 m$ tuity transformation which uses the rotaton mally $R$ In addiluon, when required, $R(p, q)$ denotes the simulanty transtormation which zeroes clements $A(p, q)$ and its symonctio 


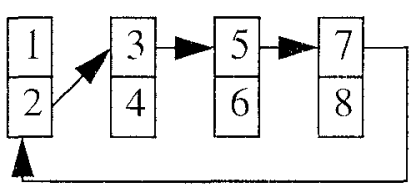

(a)

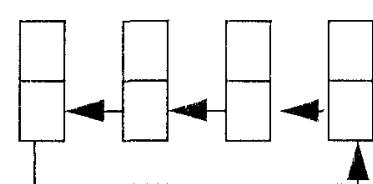

(b) step

$1 \quad(1.2)(3,4)(5,6)(7,8)$

$2 \quad(1.7)(2.4)(3.6)(5.8)$

$3(1.4)(2.6)(3.8)(5.7)$

$+\quad(1.5)(4.6)(2.8)(3.7)$

$5 \quad(1,6)(4.8)(2.7)(3.5)$

$6 \quad(1.3)(6.8)(4.7)(2.5)$

$7 \quad(1.8)(6.7)(4.5)(2.3)$

(c) step

$1 \quad(1,2)(8,7)(6,5)(4.3)$

$2(1,4)(2,7)(8,5)(6,3)$

$3(1,7)(2,5)(8,3)(6,4)$

$4 \quad(1.6)(7,5)(2,3)(8.4)$

$5 \quad(1,5)(7,3)(2,4)(8,6)$

$6 \quad(1,8)(5,3)(7,4)(2,6)$

$7 \quad(1.3)(5,4)(7.6)(2,8)$

(d)

Figure 1: Column exchange in the 1D algorithm (a) Pattern exchange for odd steps (b) Pattern exchange for even steps. (c) Column pairing for odd sweeps (d) Column pairing for even sweeps.

$$
\begin{aligned}
& A_{k}(p, q)=\left\langle U_{k}\left(^{*}, p\right), \bar{A}_{k}(*, q),>\right. \\
& A_{k}(p, p)=\left\langle U_{k}\left({ }^{*}, p\right), \bar{A}_{k}\left({ }^{*}, p\right),>\right. \\
& A_{k}(q, q)=<U_{k}\left(^{*}, q\right), \bar{A}_{k}\left(*^{*}, q\right),>
\end{aligned}
$$

where $\langle x, y\rangle$ denotes the inner product of vectors $x$ and $y$ and $\bar{A}\left({ }^{*}, p\right)$ denotes column $p$ of $\bar{A}$. If the eigenvector are to be computed ${ }^{1}$, as assumed in our paper, both one-sided and two-sided methods require the same amount of computation. On the other hand. both recovering the elements of $A$ and applying the transformation involve only column operations, which is a very interesting property for parallel distributed memory implementation Because of this potential benefit in a parallel enviromment. we focus on the one-sided Jacobi method

\subsection{Parallelism in Jacobi methods}

An interesting property of jacobi methods is that several transformations can be applied in parallel to zero several elements, reducing in this way the time required to complete a sweep. For example, elements $A(1,2)$ and $A(3,4)$ (and their symmetrics) can be zeroed in parallel because transformation $R(1,2)$ updates only columns 1 and 2 of $A$ and transformation $R(3,4)$ updates only columns 3 and 4 . For this reason, these transformations are said to be independent transformations. In general, two transformations $R(i, j)$ and $R(r ; s)$ are independent if $i \neq r, \quad \neq s, j \neq r$, and $j \neq s$. This feature has motivated the proposal of parallel Jacobi orderings in which the similarity transformations required to complete a sweep are organized into groups of independent transformations. Each of these groups will be called a step Parallel algorithms using such Jacobi orderengs can exploit the parallelism provided by a multicomputer since the work associated with one step can be distributed among the nodes in the system.

Parallel Jacobi algorithms have been proposed for different parallel computing scenarios (systolic arrays, shared memory multiprocessors. hypercube multıcomputers, mesh-connected multicomputers). In the

1. Note that in the one-sided inethod, the eIgenvectors atc the columns of $U_{\infty}$ particular case of mosh-connected multicomputers, the one-dimensional organization of the nodes (either as a line or as a rung) has been the most trequent assumption.

\subsection{A one-dimensional algorithm}

In this section we describe in detail a parallel algorithm which uses one of the Jacobi orderings proposed in [4]. This parallel algorithm will be referred to as onedimensional (1D) sunce $t 1$ uses a one-dimensional organization of the nodes. The ID algorithm will be used here to motivate our proposal and for comparison purposes.

The ID algorithm unes 2 nodes arranged as a ring (a line with a wraparound link) The algorithm is first described assumme that $m=2^{i+1}$ Intually, every node stores two columns of $\bar{\lambda}_{0}=A$ and the corresponding columns of $U_{0}=1$ Figure la shows this initial column distribution, for the particular case of $2^{d t}=4$ (columns of $\bar{A}$ and $U$ are numbered from 1 to $n l$ )

Every sweep has $2^{1 /+1}-1$ steps, each of them consisting of $m / 2$ indepondent transformations. In every step, each mole performs one of the independent transformations and exchanges one column of $\bar{A}$ and the corresponding column of $U$, with one of the nodes of the ring. This column exchange is carried out according to the patterns shown in figure la (for odd steps) and figure 16 (for even steps). Figure lc shows the pairs of columns that constitute every step in the first sweep. In this figure, a parr $(i, j)$ indicates that the curresponding node. say $q$, computes in that step the rotation $R(t, j)$ and updates $\bar{A}$ and $U$. The one-sided approach guarantees that the only data required to perform this computatron are columns $i$ and $j$ of matrices $\bar{A}$ and $U$, which reside in node q during the step. Figure Id shows the pairs which constitute every step in the second sweep. In general, odd swceps are als in figure $1 \mathrm{c}$ and even sweeps are as in figure $1 \mathrm{~d}$.

The extension of the ID algorithm to an arbitrary matrix of saze $m$ is stralghtforward. The columns of matrices $\bar{T}_{()}$and $U_{0}$ are organized into $2^{d+1}$ blocks of $m / 2^{d+1}$ consecutrve columns each Now, an index $t$ identifies a block of columns and the parr $(i, j)$ identifies the transformations whach anvolve the columns of blocks $;$ 
and $j$. In each one of the $2^{d+1}-1$ steps, every node must perform $m / 2^{d+1} \times m / 2^{d+1}$ transformations corresponding to the pairing of every column of block $i$ with all the columns of block $j$. In the particular case of the first step, the nodes perform $m / 2^{d+1} \times\left(m / 2^{d+1}-1\right)$ additional transformations corresponding to the pairing of every column with all the columns in its own block. Finally, the exchanges at the end of every step now involve blocks of columns instead of single columns.

The ID algorithm has two interesting properties related to the efficiency of a parallel implementation. First, the ordering uses a minimum number of steps. Second, it requires that at every step, each node only exchanges one block of matrices $\bar{A}$ and $U$. This is in contrast to other orderings such as round-robin [2], which require that almost all nodes exchange both blocks of $\bar{A}$ and $U$ at every step.

Consider now the problem of executing the ID algorithm on the target scenario. The straightforward approach is to make $r=0$ and $c=d$. This configures the mesh as a line, matching the one-dimensional topology assumed by the algorithm. In what follows, we develop a simple analytical model to assess the performance of this approach.

First note that computing a rotation matrix requires $6 \mathrm{~m}$ operations (we count only the operations required for inner products), and applying it requires $12 \mathrm{~m}$ operations. Second, note that all the column exchanges required in the odd steps (figure 1a) and even steps (figure 1b) can be carried out in the wormhole line simultaneously without conflicts in the use of the links. Therefore, the time required for the column exchange at every step is:

$$
t_{s}+\frac{m^{2}}{2^{d}} \times t_{c}
$$

As a result, the time required to complete one sweep can be written as:

$$
t_{1 D}=\frac{9 m^{3}-9 m^{2}}{2^{d}} \times t_{c}+\left(2^{d+1}-1\right)\left(t_{s}+\frac{m^{2}}{2^{d}} \times t_{c}\right)
$$

Expression (1) shows that the communication cost grows as $2^{d+1}-1$. This fact may degrade the performance of the ID algorithm for large values of $d$.

Note that alternative configurations of the target scenario (i.e. other values for $r$ and $c$ ) would not reduce the communication cost of the one-dimensional algorithm since the number of steps would still be $2^{d+1}-1$.

\section{Two-dimensional algorithm}

\subsection{Motivation}

The two-dimensional (2D) algorithm proposed here is aimed at reducing the communication cost incurred by the ID algorithm on a line. The $2 \mathrm{D}$ algorithm uses a $2^{r} \times 2^{c}$ mesh of nodes. The computation is organized in such a way that the transformations that were applied independently by a group of consecutive nodes in the line

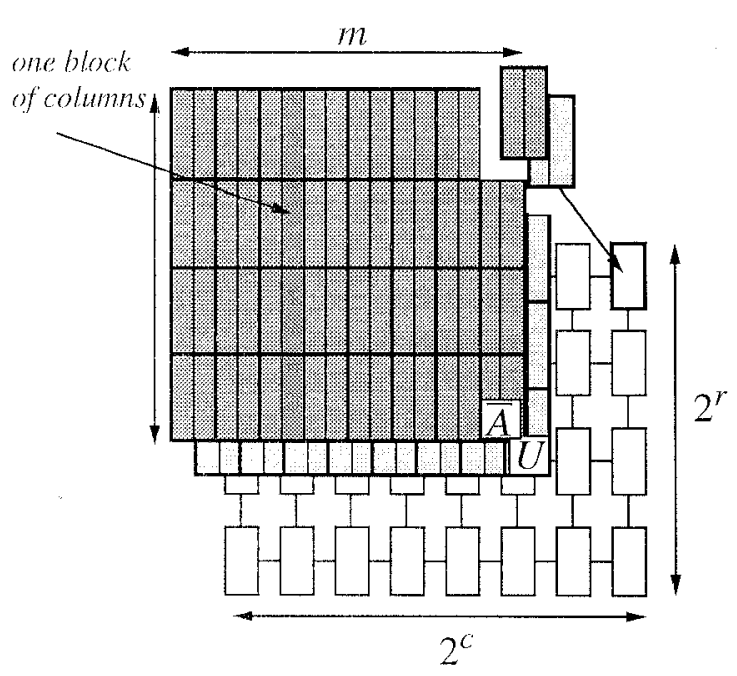

Figure 2: Data distribution in the 2D algorithm

are now applied by a column of nodes in the $2 \mathrm{D}$ mesh. Since the number of steps per sweep depends on the number of columns in the mesh $\left(2^{\circ}\right)$, a $2 \mathrm{D}$ algorithm with $c<d$ would have less steps than the ID algorithm. This could result in potentially lower cost due to column exchanges. From now on, the column exchanges at the end of every step will be referred to as horizontal communication. However, since the nodes in a column must cooperate to compute and apply the transformations, a new type of communication appears. This will be referred to as vertical communication and its cost depends on the number of rows in the mesh $\left(2^{\prime}\right)$. It will be shown that a proper choice for $r$ and $c$ can reduce significantly the communication cost (horizontal communication plus vertical communication) with respect to the $1 \mathrm{D}$ algorithm. In the following, we describe in detail the proposed $2 \mathrm{D}$ algorithm.

\subsection{Data and computation distribution}

The data distribution required by the $2 \mathrm{D}$ algorithm is shown in figure 2 . The initial matrices $\bar{A}_{0}$ and $U_{0}$ are decomposed into $2^{x+1}$ blocks of $m / 2^{c+1}$ consecutive columns. Every pair of blocks of $\bar{A}_{0}$ (and the corresponding blocks of $U_{0}$ ) are distributed among the $2^{r}$ nodes of a mesh column. As a result, every node stores $m / 2^{c}$ pieces of columns of $\bar{A}_{0}$ and $m / 2^{c}$ pieces of columns of $U_{0}$. Each of these pieces of columns has $m / 2^{r}$ elements.

In the 2D algorithm, each sweep consists of $2^{c+1}-1$ steps. At each step, the $2^{r}$ nodes of a column cooperate to compute and apply a total of $m / 2^{c+1} \times m / 2^{c+1}$ transformations. This corresponds to pairing every column of one block with all the columns in the other block. In the first step, $m / 2^{c+1} \times\left(m / 2^{c+1}-1\right)$ additional transformations must be computed and applied, corresponding to pairing every column with all the remaining columns in its own block.

The computation and the application of every 
transformation is now distributed among the nodes of the column, since every node stores only a piece of $m / 2^{\prime}$ elements of the two columns from $\bar{A}$ and $U$ involved in the transformation. Let us focus on transformation $R(i, j)$. The activity of the nodes in the column of the mesh responsible for computing and applying $R(i, j)$ can be organized into three phases as follows:

Phase 1. Every node in the column performs three partial inner products corresponding to the recovering of elements $A(i, j), A(i, i)$ and $A(j, j)$. To that end, every node uses the pieces of columns $i$ and $j$ of matrices $\bar{A}$ and $U$. The result is a set of three values in every node of the column.

Phase 2. The nodes in the column cooperate to accumulate the partial inner products and obtain the final values of $A(i, j), A(i, i)$ and $A(j, j)$. At the end of this phase, a copy of these three final values must be stored in every node in the column. It is in phase 2 where the vertical communication appears.

Phase 3. Every node computes $s=\sin \left(\alpha_{i, j}\right)$ and $c=\cos \left(\alpha_{i, j}\right)$ and applies the transformation to the pieces of columns $i$ and $j$.

At each step, every column of nodes must repeat phases 1, 2 and 3 for every one of the assigned transformations. However, proceeding in this way would make the cost of the vertical communication very high since the nodes would exchange a large amount of very short messages (only three elements per message). In order to amortize the communication startup cost, the transformations assigned to each mesh column at every step are organized into groups of independent transformations. Now, for each group of independent transformations, phase 1 is performed for every transformation within the group. Then, vertical communication is carried out to exchange the partial inner products corresponding to all the transformations in the group. Finally, phase 3 can be carried out for all the transformations in the group. In this way, the number of messages involved in the vertical communication is reduced since every message now contains a larger number of partial inner products.

For a given step, the problem of organizing the computation assigned to a column of the mesh into groups of independent transformations can be solved in many different ways. In fact, the approaches to obtain parallel Jacobi orderings can be readily applied to solve this problem since the objective is exactly the same, that is, to find groups of independent transformations. In general, in the first step of every sweep, every column of the mesh organizes the computation into $m / 2^{c}-1$ groups of $m / 2^{c+1}$ independent transformations each. In every one of the remaining steps of a sweep, each column of the mesh works with $m / 2^{c+1}$ groups, with $m / 2^{c+1}$ independent transformations in each group.

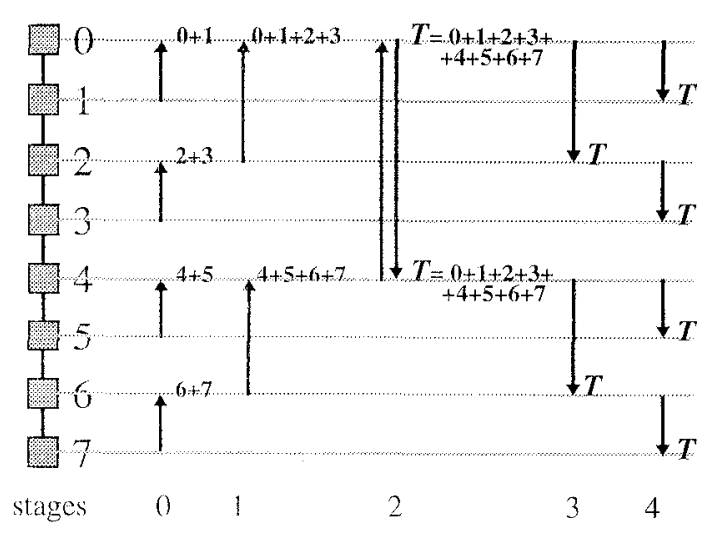

Figure 3: An example (with $r=3$ ) of the algorithm to implement the vertical communication arising when applying the transformations in a group.

\subsection{Vertical and horizontal communication}

We describe first the proposed scheme to perform the vertical communication which arises when performing a group of independent transformations. The communication problem consists of a multiple vector addition. Every one of the $2^{r}$ nodes in the column stores a vector of $3 m / 2^{c+1}$ values, corresponding to the partial inner products of the $m / 2^{c+1}$ independent transformations of the group. All these vectors must be accumulated and, at the end, every node must have a copy of the resulting vector. Figure 3 shows an example of the scheme to solve this problem assuming a wormhole line for the case of $r=3$. In this figure, the numbers identify the nodes in the column and also the vectors to be accumulated. Arrows represent communication between nodes. $T$ denotes the final vector. In the following, we describe the communication algorithm in detail. We denote by $<n_{r-1}, \ldots, n_{1}, n_{0}>$ the binary form of integer $n\left(n \in\left[0,2^{r}-1\right]\right)$.

The algorithm proceeds in $2 r-1$ stages, numbered from 0 to $2 r-2$. In stages $i$, with $i \in[0, r-2]$, only nodes with label $n$ such that $n_{j-1}=\ldots=n_{0}=0$ are active. In particular, a node with label $\left\langle n_{r-1}, \ldots, n_{i+1}, 1,0, \ldots, 0\right\rangle$ sends its partial vector to the node with label $\left.<n_{r-1}, \ldots, n_{i+1}, 0,0, \ldots, 0\right\rangle$, which, after receiving the messages, accumulates the received vector within its own partial vector. In stage $r-1$, nodes $2^{r-1}$ and 0 exchange a copy of their partial vectors and accumulate the received vector. At this time, both nodes, $2^{r-1}$ and 0 , have a copy of the final vector $T$. Finally, in stages $2 r-2-i$, with $i \in[0, r-2]$, the node with label $\left.<n_{i-1}, \ldots, n_{i+1}, 0,0, \ldots, 0\right\rangle$ sends a copy of the final vector to the node with label $<n_{r-1}, \ldots, n_{i+1}, 1,0, \ldots, 0>$

We now focus on the horizontal communication that is required at the end of every one of the $2^{c+1}-1$ steps of a sweep. In this communication operation, the columns of matrices $\bar{A}$ and $U$ are exchanged so that every node receives the pieces of the columns required to perform the transformations in the next step. Every process 


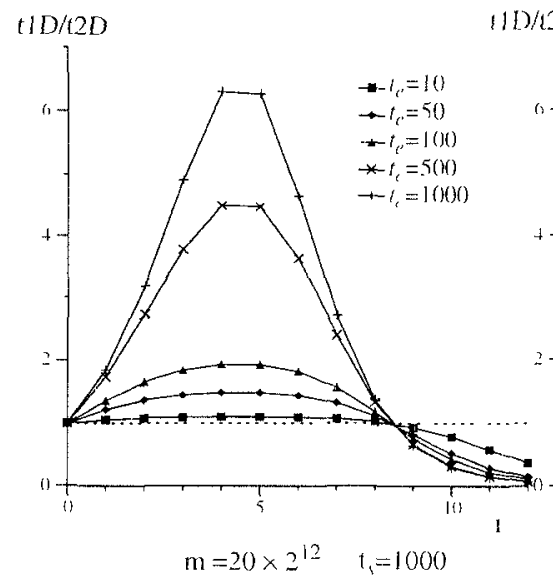

(a)

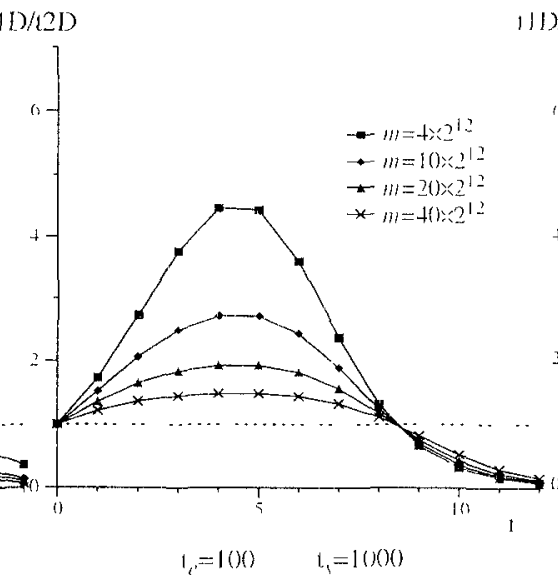

(b)

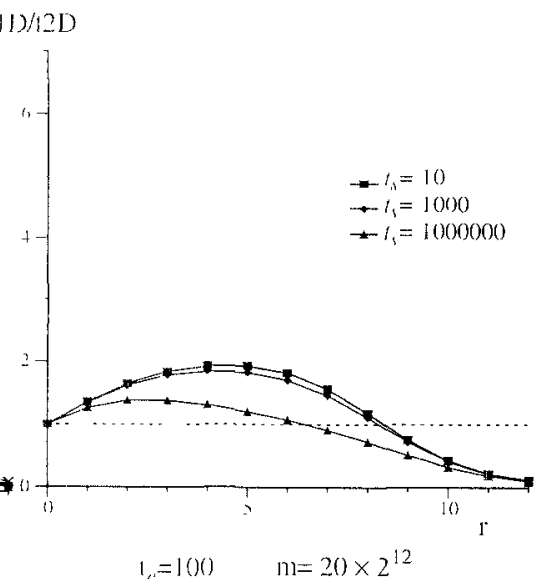

(c)

Figure 4: Impact of parameters $t_{e}, m$ and $t_{s}$ in the performance improvement of the proposed $2 \mathrm{D}$ algorithm

partrcipates in a communication in the horizontal dimension of the mesh, according to the same exchange patterns that are used by the 1D algorithm (and shown in figures $l a$ and $l b$ ). In particular, nodes exchange messages of $m^{2} / 2^{d}$ elements, corresponding to the pieces of columns in one block of $\bar{A}$ and the corresponding block of $U$.

\section{Analytical modelling and evaluation of the 2D algorithm}

\subsection{An analytical model}

It is obvious that increasing the value of $r$ (and as a result decreasing c) reduces the number of steps of the $2 \mathrm{D}$ algorithm and therefore, the cost of the horizontal communication, at the expense of an increase in the cost of vertical communication. The analytical model of execution time that will be developed in this section, will enable us to derive the optimal configuration of the mesh. This would correspond to the values of $r$ and $c$ which minimizes the execution time.

We consider first the cost of the partial inner computation and the application of the transformation (phases 1 and 3, described in section 3.2).This cost is:

$$
t_{R}=(m-1) \times \frac{m}{2^{c+1}} \times \frac{18 m}{2^{\prime}} \times t_{1}=\frac{9 m^{3}-9 m^{2}}{2^{d}} \times t_{1}
$$

The term $m-1$ corresponds to the number of groups of independent transformations in a sweep. the term $m / 2^{i+1}$ is the number of independent transformations per group, and the term $18 \mathrm{~m} / 2^{\prime}$ is the number of operations to compute the partial inner product (phase 1) and apply (phase 3) the transformation.

The cost of the vertical communication (including the cost of partial vector accumulation) is given by the following expression:

$$
\begin{gathered}
l_{1}=(m-1)(21-1)\left(1+\frac{3 m}{2^{1+1}} \times t_{c^{\prime}}\right)+ \\
(m-1)(1-1)\left(\frac{3 m}{2^{c+1}} \times t_{c}\right)
\end{gathered}
$$

This expression is obtamed by considering again the number of groups of independent transformation in a sweep ( $m-1$ ) and the cost of the vertical communication per group, according to the algorithm proposed in section 3.3

The cost of the horizontal communication is written as follows:

$$
t_{H}=\left(2^{t+1}-1\right)\left(t_{4}+\frac{m^{2}}{2^{l}} \times t_{e}\right)
$$

Finally, the total cost of the algorithm is $t_{2 D}=t_{R}+t_{V}$ $+t_{H}$. Although it is not possible to write a simple analytical expression for the optimal values of $c$ and $r$, these values can be computed by inspection, when fixing the values for the rest of the parameters of the analytical model

\subsection{Performance evaluation}

In this sectron, we plot some figures to show the improvement in performance that can be obtained with the 2D algorithm, with respect to the ID algorithm. These figures have been obtanned from the analytical models of $t_{1 D}$ and $t_{2 D}$ derived in the previous sections.

The figures are presented in two types of plots. Plots in figure 4 assume a fixed value of $d$ (specifically $d=12$ ) and show the ratio $t_{10} / t_{21}$, for all possible values of $r(r$ $\in[0, d])$ and varying values of parameters $t_{c}$ (figure $\left.4 \mathrm{a}\right), m$ (figure th), and $t$, (figure $4 c)(t$, is fixed at 1 ). In every plot, one of these three parameter takes different values while the remainng two are fixed, showing in this way the impact of the varying parameter on the performance 
improvement and on the optimal configuration of the mesh.

The following conclusions can be drawn from the plots in figure 4 :

- Parameter $t_{e}$ (transmission time per floating point number) has a strong impact on performance improvement (see figure 4a). This improvement can be very high for large values of $t_{e}$. On the other hand. the value of $t_{e}$ does not have an impact in the optumal configuration.

- The performance improvement is also very sensitive to the values of $m$, but in this case, large values of $m$ reduce this improvement (see figure $4 \mathrm{~b}$ ). Varying $n$ does not changes the optimal configuration.

- Large values of $t$, reduce the performance improvement and favour configurations with smaller number of rows (see figure $4 c$ ). However, these etfects are only noticeable for extremely large values of $t_{\mathrm{s}}$ Therefore, the performance improvement is not very sensitive to the value of $t_{\varsigma}$.

- The optimal configuration usually has less rows than columns, since the vertical communication cost grows very fast with the number of rows (note that in the expression of $t_{\mathrm{V}}$ the term $t_{t^{\prime}}$ is affected by $m$ ).

The plots in figure 5 show the scalability properties of the proposed algorithm. These plots show the ratio $t_{1 f} / t_{2 D}$ for fixed values of $t_{s}$ and $t_{c}$, but varying values of $d$ and $m$ (note that $m$ is now scaled with the number of nodes). In this case, for a given value of $d$, the optimal configuration in considered for $t_{2 D}$ The plots show that the performance improvement obtained with the $2 \mathrm{D}$ algorithm grows when increasing the number of nodes in the system. This growth is particularly high for small problem sizes.

\section{Summary}

The figures in section 4 show that the proposed 20 algorithm for symmetric eigenvalue and eigenvector computation can provide a significant performance improvement with respect to the $1 \mathrm{D}$ algorithm, for a wide range of system and problem parameters This improvement increases with the system size, showing that the algorithm is scalable, which is an important property when looking at large scale parallel systems.

Further work is currently focusing on assuming a mesh with a fixed configuration. and in particular, a squared mesh (a $2^{d / 2} \times 2^{d / 2}$ mesh, with $d$ even) [8]. In this case, an obvious solution is to use the algorithm proposed in this paper, with $r=c=d / 2$. However, this may not be the optimal choice. A more general approach would be to embed a $2^{r} \times 2^{\prime}$ mesh onto the squared mesh and analyse the impact of such an embedding on the cost of vertical and horizontal communication, in order to select the optimal values for $r$ and $c$.

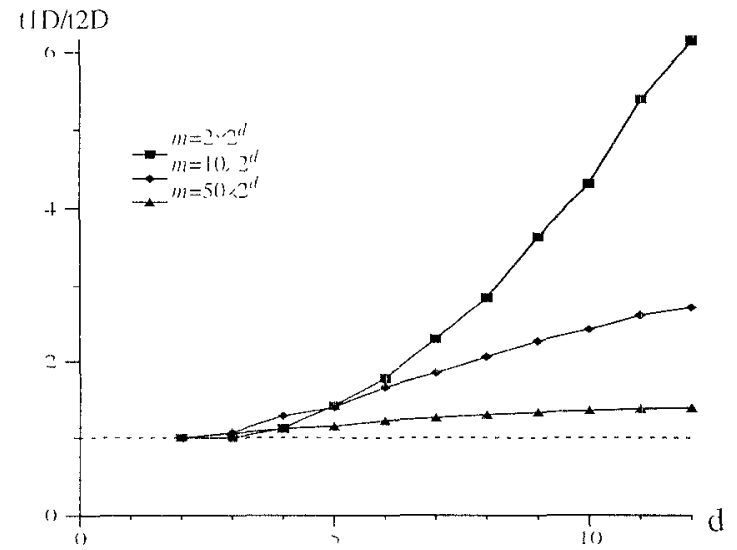

Figure 5: Performance improvement of the proposed 2D algorithm for an increasing number of nodes.

\section{References}

[1] M. Berry' and A. Sameh, "Multıprocessor Jacobi Schemes for Dense Symmetric Eigenvalue and Singular Value Decompositions," Proceedings of ICPP 98 (1986), pp. 433-44().

[2] R.P Brent and F.T. Luk, "The solution of singularvalue and syınmetric eigenvalue problems on multuprocessor a rays," SIAM J. Sci. Statist. Comput. 6 (1985), pp. 69-84.

131 P.J. Eberlein, "On one-sided Jacobi methods for parallel computation," SIAM J. Algebraic Discrete Methods 8 (1987), pp. 790-796

[4] P.J. Eberlein and H Park. "Etficrent implementation of Jacobr algorithms and Jacobi sets on distributed memory architectures," Journal of Parallel Distributed Conputing 8 (1990), pp. 358-366.

[5] G H. Golub and C.F. Van Loan, Matrix computations, Baltimore. MD: The John Hopkins Univ. Press, 1989.

[6] F.T. Luk, "Architectures for computing eigenvalues and SVDs," SPIE Vol. 614 Highly Parallel Signal Processing Arfitectues (1986). pp. 24-33.

171 L.M. Ni and PK. McKinley, "A survey of wormhole routng techniques in directed networks," IEEE Complter. Vol. 26, No 2. February 1993, pp. 62-76.

181 D. Royo, M Valero-García and A. González, "A Jacobi-like Algorithm for Eigenvalue Computation on a 2D/3D mesh multicomputer". Technical Report UPC-DAC-1997-73, Cinvertstat Politècnica de Critalunici

19] R.A Whiteside. NS Ostlund, and P.G. Hibbard. "A parallel Jacobi diagonalization algorithm for a loop multiple processor system," IEEE Trans. Comput. C33 (1984). pp $4(109-413$

[10] f H. Wilkinson. The Algebratc Eigenvalue Problem. Claredon Press, Oxford, 1965.

[11] B.B. Zhou and R.P. Brent, "A Parallel Ring Ordering Algorithm for Efficient One-Sided Jacobi SVD Computations," Jromal of Parallel and Distributed Computing (1997), 42, 1-10. 